\title{
POSITIVE SOLUTIONS FOR NONLINEAR NONHOMOGENEOUS PARAMETRIC EQUATIONS
}

\author{
Nikolaos S. Papageorgiou - George Smyrlis
}

\begin{abstract}
We consider a nonlinear parametric Dirichlet problem driven by a nonhomogeneous differential operator which includes as special cases the $p$-Laplacian, the $(p, q)$-Laplacian and the generalized $p$-mean curvature operator. Using variational methods, we prove a bifurcation-type theorem describing the dependence of positive solutions on the parameter.
\end{abstract}

\section{Introduction}

Let $\Omega \subseteq \mathbb{R}^{N}$ be a bounded domain with a $C^{2}$-boundary $\partial \Omega$. In this paper, we study the following nonlinear Dirichlet eigenvalue problem

$(\mathrm{P})_{\lambda} \quad-\operatorname{div} a(D u(z))=\lambda f(z, u(z)) \quad$ in $\Omega,\left.\quad u\right|_{\partial \Omega}=0, \quad u>0, \quad \lambda>0$.

In $(\mathrm{P})_{\lambda}$ the map $a: \mathbb{R}^{N} \rightarrow \mathbb{R}^{N}$ is strictly monotone and satisfies certain other regularity conditions. The precise conditions on $a(\cdot)$ are stated in hypotheses $\mathrm{H}(\mathrm{a})$ below. They provide a unifying framework to treat equations driven by the $p$-Laplacian, the $(p, q)$-Laplacian differential operator and the generalized $p$-mean curvature differential operator. Also, $\lambda>0$ is a parameter and $f: \Omega \times \mathbb{R} \rightarrow \mathbb{R}$ is a Caratheodory function (i.e. for all $x \in \mathbb{R}, z \rightarrow f(z, x)$ is measurable and for almost all $z \in \Omega, x \rightarrow f(z, x)$ is continuous), which is strictly $(p-1)$-sublinear in the $x$-variable near $+\infty$. We prove a bifurcation-type result describing precisely the dependence of positive solutions of $(\mathrm{P})_{\lambda}$ on the parameter $\lambda>0$. Recently

2010 Mathematics Subject Classification. 35J20, 35J25, 35J60, 35J92.

Key words and phrases. Nonlinear regularity, mountain pass theorem, bifurcation-type theorem, strong comparison principle, nonhomogeneous differential operator, positive solutions. 
positive solutions for nonlinear eigenvalue problems driven by the $p$-Laplacian, were obtained by Brock, Itturiaga and Ubilla [5], $\mathrm{Hu}$ and Papageorgiou [13] and Perera [18]. In contrast to our differential operator here, the $p$-Laplacian is $(p-1)$-homogeneous and this is a feature that helps the analysis of the equation. Finally we should mention the recent work of Cardinali, Papageorgiou, Rubbioni [6], where an analogous result was proved for a Neumann logistic equation driven by the $p$-Laplacian.

\section{Hypotheses - auxiliary results}

Let $\vartheta \in C^{1}(0, \infty)$ be such that

$$
\begin{array}{cc}
0<\widehat{c} \leq \frac{t \vartheta^{\prime}(t)}{\vartheta(t)} \leq c_{0} & \text { for all } t>0, \\
c_{1} t^{p-1} \leq \vartheta(t) \leq c_{2}\left(t^{q-1}+t^{p-1}\right) & \text { for all } t>0
\end{array}
$$

and some $c_{1}, c_{2}>0,1<q<p$. The hypotheses on the map $a(\cdot)$ are the following:

$\mathrm{H}(\mathrm{a}) a(y)=a_{0}(\|y\|) y$ for all $y \in \mathbb{R}^{N}$ with $a_{0}(t)>0$ for all $t>0, a(0)=0$ and

(i) $a_{0} \in C^{1}(\mathbb{R} \backslash\{0\}) \cap C(\mathbb{R})$ and $\lim _{s \rightarrow 0^{+}} \frac{s a^{\prime}(s)}{a(s)}>-1$;

(ii) $\|\nabla a(y)\| \leq c_{3} \frac{\vartheta(\|y\|)}{\|y\|}$ for all $y \in \mathbb{R}^{N} \backslash\{0\}$ and some $c_{3}>0$;

(iii) $(\nabla a(y) \xi, \xi)_{\mathbb{R}^{N}} \geq \frac{\vartheta(\|y\|)}{\|y\|}\|\xi\|^{2}$ for all $y \in \mathbb{R}^{N} \backslash\{0\}$, all $\xi \in \mathbb{R}^{N}$.

REMARK 2.1. Let

$$
G_{0}(t)=\int_{0}^{t} a_{0}(s) s d s, \quad t \geq 0 .
$$

Evidently $G_{0}(\cdot)$ is strictly convex and strictly increasing. For all $y \in \mathbb{R}^{N}$ we set $G(y)=G_{0}(\|y\|)$. Then $G(\cdot)$ is convex, $G(0)=0$ and for all $y \in \mathbb{R}^{N} \backslash\{0\}$, we have

$$
\nabla G(y)=G_{0}^{\prime}(\|y\|) \frac{y}{\|y\|}=a_{0}(\|y\|) y=a(y) .
$$

Hence $G(\cdot)$ is the primitive of $a(\cdot)$. Since $G(\cdot)$ is convex and $G(0)=0$, we have

$$
G(y) \leq(a(y), y)_{\mathbb{R}^{N}} \quad \text { for all } y \in \mathbb{R}^{N} .
$$

From hypotheses $\mathrm{H}(\mathrm{a})$ and $(2.2),(2.3)$, we easily deduce the following properties of the map $a(\cdot)$.

LEMmA 2.2. If hypotheses $\mathrm{H}(\mathrm{a})$ hold, then:

(a) $y \rightarrow a(y)$ is maximal monotone and strictly monotone;

(b) $\|a(y)\| \leq c_{4}\left(1+\|y\|^{p-1}\right)$ for all $y \in \mathbb{R}^{N}$ and some $c_{4}>0$;

(c) $(a(y), y)_{\mathbb{R}^{N}} \geq c_{1}\|y\|^{p} /(p-1)$ for all $y \in \mathbb{R}^{N}$ (see $(2.2)$ ). 
From this lemma and the integral form of the mean value theorem, we obtain the following growth conditions on $G(\cdot)$.

Corollary 2.3. If hypotheses $\mathrm{H}(\mathrm{a})$ hold, then $\frac{c_{1}}{p(p-1)}\|y\|^{p} \leq G(y) \leq c_{5}\left(1+\|y\|^{p}\right) \quad$ for all $y \in \mathbb{R}^{N}$ and some $c_{5}>0$.

EXAMPLES 2.4. The following maps satisfy hypotheses H(a):

(a) $a(y)=\|y\|^{p-2} y$ with $1<p<\infty$.

This map corresponds to the $p$-Laplace differential operator

$$
\Delta_{p} u=\operatorname{div}\left(\|D u\|^{p-2} D u\right) \quad \text { for all } u \in W_{0}^{1, p}(\Omega) .
$$

(b) $a(y)=\|y\|^{p-2} y+\|y\|^{q-2} y$ with $1<q<p<\infty$.

This map corresponds to the $(p, q)$-differential operator

$$
\Delta_{p} u+\Delta_{q} u \text { for all } u \in W_{0}^{1, p}(\Omega) .
$$

This differential operator is important in quantum physics (see Benci, D'Avenia, Fortunato and Pisani [3]) and in reaction diffusion equations and plasma physics (see Cherfils and Ilyasov [7]). Recently such equations were studied by Cingolani and Degiovanni [8], Li and Guo [16], Sun [20].

(c) $a(y)=\left(1+\|y\|^{2}\right)^{(p-2) / 2} y$ with $1<p<\infty$.

This map corresponds to the generalized $p$-mean curvature differential operator

$$
\operatorname{div}\left(\left(1+\|D u\|^{2}\right)^{(p-2) / 2} D u\right) \quad \text { for all } u \in W_{0}^{1, p}(\Omega) .
$$

Such equations can be found in Pucci and Serrin [19].

(d) $a(y)=\|y\|^{p-2} y+\|y\|^{p-2} y /\left(1+\|y\|^{p}\right)$ with $1<p<\infty$.

(e) $a(y)=\|y\|^{p-2} y+\ln \left(1+\|y\|^{p}\right) y \quad$ with $1<p<\infty$.

Let $f_{0}: \Omega \times \mathbb{R} \rightarrow \mathbb{R}$ be a Caratheodory function such that

$$
\left|f_{0}(z, x)\right| \leq \alpha(z)+c|x|^{r-1} \quad \text { for a.a. } z \in \Omega \text {, all } x \in \mathbb{R},
$$

with $\alpha \in L^{\infty}(\Omega)_{+}, c>0$ and

$$
1<r<p^{*}= \begin{cases}\frac{N p}{N-p} & \text { if } p<N \\ +\infty & \text { if } p \geq N .\end{cases}
$$

We set

$$
F_{0}(z, x)=\int_{0}^{x} f_{0}(z, s) d s
$$


and consider the $C^{1}$-functional $\varphi_{0}: W_{0}^{1, p}(\Omega) \rightarrow \mathbb{R}$ defined by

$$
\varphi_{0}(u)=\int_{\Omega} G(D u(z)) d z-\int_{\Omega} F_{0}(z, u(z)) d z \quad \text { for all } u \in W_{0}^{1, p}(\Omega) .
$$

The next result can be found in Gasinski and Papageorgiou [13].

Proposition 2.5. If $u_{0} \in W_{0}^{1, p}(\Omega)$ is a local $C_{0}^{1}(\bar{\Omega})$-minimizer of $\varphi_{0}$, i.e. there exists $\rho_{0}>0$ such that

$$
\varphi_{0}\left(u_{0}\right) \leq \varphi_{0}\left(u_{0}+h\right) \quad \text { for all } h \in C_{0}^{1}(\bar{\Omega}), \quad\|h\|_{C_{0}^{1}(\bar{\Omega})} \leq \rho_{0},
$$

then $u_{0} \in C^{1, \beta}(\bar{\Omega})$ for some $\beta \in(0,1)$ and $u_{0}$ is also a local $W_{0}^{1, p}(\Omega)$-minimizer of $\varphi_{0}$, i.e. there exists $\rho_{1}>0$ such that

$$
\varphi_{0}\left(u_{0}\right) \leq \varphi_{0}\left(u_{0}+h\right) \quad \text { for all } h \in W_{0}^{1, p}(\Omega), \quad\|h\| \leq \rho_{1} .
$$

REMarK 2.6. The first such result was proved by Brezis and Nirenberg [4] for the case when $G(y)=\|y\|^{2} / 2$ for all $y \in \mathbb{R}^{N}$. It was extended to the case $G(y)=\|y\|^{p} / p$ for all $y \in \mathbb{R}^{N}$ with $1<p<\infty$ by Garcia Azorero, Manfredi and Peral Alonso [9]. The proof of [13] differs from the proofs in [4], [9].

In the analysis of problem $(\mathrm{P})_{\lambda}$, we will use the ordered Banach space

$$
C_{0}^{1}(\bar{\Omega})=\left\{u \in C^{1}(\bar{\Omega}):\left.u\right|_{\partial \Omega}=0\right\} .
$$

The order cone of this space is

$$
C_{+}=\left\{u \in C_{0}^{1}(\bar{\Omega}): u(z) \geq 0, \text { for all } z \in \bar{\Omega}\right\} .
$$

This cone has a nonempty interior given by

$$
\operatorname{int} C_{+}=\left\{u \in C_{+}: u(z)>0 \text { for all } z \in \Omega, \frac{\partial u}{\partial n}(z)<0 \text { for all } z \in \partial \Omega\right\},
$$

where $n(\cdot)$ is the outward unit normal on $\partial \Omega$.

Also throughout this work by $\|\cdot\|$ we denote the norm of the Sobolev space $W_{0}^{1, p}(\Omega)$. By virtue of the Poincare inequality, we have $\|u\|=\|D u\|_{p}$ for all $u \in W_{0}^{1, p}(\Omega)$. By $|\cdot|_{N}$ we denote the Lebesgue measure on $\mathbb{R}^{N}$. For $x \in \mathbb{R}$, we set $x^{ \pm}=\max \{ \pm x, 0\}$. If $u \in W_{0}^{1, p}(\Omega)$ then

$$
u^{ \pm}(\cdot)=u(\cdot)^{ \pm} \in W_{0}^{1, p}(\Omega) \quad \text { and } \quad|u|=u^{+}+u^{-}, \quad u=u^{+}-u^{-} .
$$

If $h: \Omega \times \mathbb{R} \rightarrow \mathbb{R}$ is a measurable function (for example a Carathéodory function), then

$$
N_{h}(u)(\cdot)=h(\cdot u(\cdot)) \quad \text { for all } u \in W_{0}^{1, p}(\Omega) .
$$

Let $A: W_{0}^{1, p}(\Omega) \rightarrow W^{-1, p^{\prime}}(\Omega)=W_{0}^{1, p}(\Omega)^{*}\left(1 / p+1 / p^{\prime}=1\right)$ be the nonlinear map defined by

$$
\langle A(u), y\rangle=\int_{\Omega}(a(D u), D y)_{\mathbb{R}^{N}} d z \quad \text { for all } u, y \in W_{0}^{1, p}(\Omega) .
$$


Here by $\langle\cdot, \cdot\rangle$ we denote the duality brackets for the pair $\left(W_{0}^{1, p}(\Omega), W^{-1, p^{\prime}}(\Omega)\right)$.

From Gasinski and Papageorgiou [10], we have

Proposition 2.7. If hypotheses $\mathrm{H}(\mathrm{a})$ hold and $A: W_{0}^{1, p}(\Omega) \rightarrow W^{-1, p^{\prime}}(\Omega)$ is the nonlinear map defined by (2.3), then $A$ is monotone continuous bounded (i.e. maps bounded sets to bounded sets) hence maximal monotone too and of type $(S)_{+}$, i.e. if $u_{n} \stackrel{w}{\longrightarrow} u$ in $W_{0}^{1, p}(\Omega)$ and $\limsup _{n \rightarrow+\infty}\left\langle A\left(u_{n}\right), u_{n}-u\right\rangle \leq 0$, then $u_{n} \rightarrow u$ in $W_{0}^{1, p}(\Omega)$.

Let $h_{1}, h_{2} \in L^{\infty}(\Omega)$. We write $h_{1} \prec h_{2}$, if for any $K \subseteq \Omega$ compact, we can find $\varepsilon>0$ suth that

$$
h_{1}(z)+\varepsilon \leq h_{2}(z) \text { for a.a. } z \in K \text {. }
$$

Evidently, if $h_{1}, h_{2} \in C(\Omega)$ and $h_{1}(z)<h_{2}(z)$ for all $z \in \Omega$, then $h_{1} \prec h_{2}$.

The next strong comparison principle extends Proposition 2.6 of Arcoya and Ruiz [2] which was proved for the particular case of the $p$-Laplacian.

Proposition 2.8. If $\xi \geq 0, h_{1}, h_{2} \in L^{\infty}(\Omega), h_{1} \prec h_{2}$ and $u \in C_{0}^{1}(\bar{\Omega})$, $v \in \operatorname{int} C_{+}$are solutions of

$$
\begin{aligned}
-\operatorname{div} a(D u(z))+\xi|u(z)|^{p-2} u(z) * & =h_{1}(z) \quad \text { in } \Omega, \\
-\operatorname{div} a(D v(z))+\xi|v(z)|^{p-2} v(z) & =h_{2}(z) \quad \text { in } \Omega,
\end{aligned}
$$

then $v-u \in \operatorname{int} C_{+}$.

Proof. We have

$$
A(u)+\xi|u|^{p-2} u \leq A(v)+\xi|v|^{p-2} v \quad \text { in } W^{-1, p^{\prime}}(\Omega) .
$$

Acting with $(u-v)^{+} \in W_{0}^{1, p}(\Omega)$, we obtain

$$
\begin{aligned}
\left\langle A(u)-A(v),(u-v)^{+}\right\rangle & +\int_{\Omega} \xi\left(|u|^{p-2} u-|v|^{p-2} v\right)(u-v)^{+} d z \leq 0, \\
\Rightarrow & \int_{\{u>v\}}(a(D u)-a(D v), D u-D v)_{\mathbb{R}^{N}} d z \\
& +\int_{\{u>v\}} \xi\left(|u|^{p-2} u-|v|^{p-2} v\right)(u-v) d z \leq 0, \\
\Rightarrow & |\{u>v\}|_{N}=0, \quad \text { hence } u \leq v \quad \text { (see Lemma 2.2). }
\end{aligned}
$$

Next we show that $u(z)<v(z)$ for all $z \in \Omega$. To this end, we introduce the sets

$$
E_{0}=\{z \in \Omega: u(z)=v(z)\} \quad \text { and } \quad E=\{z \in \Omega: D u(z)=D v(z)=0\} .
$$

Claim. $E_{0} \subseteq E$. 
Let $z_{0} \in E_{0}$. Then the function $w=v-u$ attains its minimum at $z_{0}$ and so $D u\left(z_{0}\right)=D v\left(z_{0}\right)$. If $D u\left(z_{0}\right) \neq 0$, then we can find $\rho>0$ small such that $B_{\rho}\left(z_{0}\right) \subseteq \Omega$ and

$$
\|D u(z)\|>0, \quad\|D v(z)\|>0, \quad(D u(z), D v(z))_{\mathbb{R}^{N}}>0 \quad \text { for all } z \in B_{\rho}\left(z_{0}\right) .
$$

We have $w=v-u \in C_{+} \backslash\{0\}$ and $w(\cdot)$ satisfies the following linear elliptic equation in $B_{\rho}\left(z_{0}\right)$ (see Arcoya and Ruiz [2]):

$$
-\sum_{i, j=1}^{N} \frac{\partial}{\partial z_{i}}\left(\vartheta_{i j}(z) \frac{\partial w}{\partial z_{j}}\right)=-\xi\left(|v|^{p-2} v-|u|^{p-2} u\right)+h_{2}-h_{1} \quad \text { in } B_{\rho}\left(z_{0}\right)
$$

In $(2.4)$ the coefficients $\vartheta_{i j}(\cdot)$ are given by

$$
\vartheta_{i j}(z)=\int_{0}^{1} \frac{\partial a_{i}}{\partial y_{j}}((1-t) D u(z)+t D v(z)) d t .
$$

We have $\vartheta_{i j} \in C\left(\bar{B}_{\rho}\left(z_{0}\right)\right)$ and by choosing $\rho>0$ even smaller if necessary in $(2.4)$, we can have the differential operator uniformly elliptic and the forcing term (i.e. right hand side) positive. Then the maximum principle of Vazquez [21] implies that $u(z)<v(z)$ for all $z \in \bar{B}_{\rho}\left(z_{0}\right)$, which contradicts the fact that $z_{0} \in E_{0}$. This proves the Claim.

Since $v \in \operatorname{int} C_{+}$, we have that $E$ is compact and $E_{0}$ being a closed subset of $E$ (see the Claim), itself is also compact. Therefore we can find $\Omega_{1} \subseteq \Omega$ a smooth open set such that

$$
E_{0} \subseteq \Omega_{1} \subseteq \bar{\Omega}_{1} \subseteq \Omega
$$

We can find $\varepsilon \in(0,1)$ such that

$$
u(z)+\varepsilon \leq v(z) \text { for all } z \in \partial \Omega_{1} \quad \text { and } \quad h_{1}(z)+\varepsilon \leq h_{2}(z)
$$

for almost all $z \in \Omega_{1}$.

We choose $\delta \in(0, \varepsilon)$ small such that

$$
\left.\xi|| s\right|^{p-2} s-\left|s^{\prime}\right|^{p-2} s^{\prime} \mid \leq \varepsilon
$$

for all $s, s^{\prime} \in\left[-\|v\|_{\infty},\|u\|_{\infty}\right]$, with $\left|s-s^{\prime}\right| \leq \delta$. Then we have

$$
\begin{aligned}
& -\operatorname{div} a(D(u+\delta))+\xi|u+\delta|^{p-2}(u+\delta)=-\operatorname{div} a(D u)+\xi|u+\delta|^{p-2}(u+\delta) \\
& =\xi\left[|u+\delta|^{p-2}(u+\delta)-|u|^{p-2} u\right]+h_{1} \\
& \leq h_{1}+\varepsilon \leq h_{2} \quad(\text { see }(2.5) \text { and }(3.3)) \\
& =-\operatorname{div} a(D v)+\xi|v|^{p-2} v \quad \text { in } \Omega_{1} \text {, } \\
& \Rightarrow u+\delta \leq v \quad \text { in } \Omega_{1} \quad \text { (see Pucci, Serrin [19]). }
\end{aligned}
$$

Since $E_{0} \subseteq \Omega_{1}$, it follows that $E_{0}=\emptyset$ and so $u(z)<v(z)$ for all $z \in \Omega$. 
Next, let $z_{0} \in \partial \Omega$. Since $\partial \Omega$ is by hypothesis a $C^{2}$-manifold, we can find $\rho>0$ small such that

$$
B_{2 \rho}(\widehat{z}) \subseteq \Omega \quad \text { and } \quad z_{0} \in \partial B_{2 \rho}(\widehat{z}) \cap \partial \Omega \quad(\text { with } \widehat{z} \in \Omega)
$$

Invoking Lemma 2 of Lewis [15], we can find $\widehat{w} \in C^{1}\left(B_{2 \rho}(\widehat{z})\right)$ such that

$$
\begin{array}{rlrl}
-\operatorname{div}(\Theta(z) D \widehat{w}(z)) & =0 & & \text { in } B_{2 \rho}(\widehat{z}) \backslash \bar{B}_{\rho}(\widehat{z})\left(\Theta(z)=\left(\vartheta_{i j}(z)\right)_{i, j=1}^{N}\right), \\
\left.\widehat{w}\right|_{\partial B_{\rho}(\widehat{z})} & =1, & \left.\widehat{w}\right|_{\partial B_{2 \rho}(\widehat{z})}=0,0<\widehat{w}<1 \text { in } B_{2 \rho}(\widehat{z}) \backslash \bar{B}_{\rho}(\widehat{z}),
\end{array}
$$

and $\|D \widehat{w}(z)\| \geq \widehat{c}>0$ for all $z \in B_{2 \rho}(\widehat{z}) \backslash \bar{B}_{\rho}(\widehat{z})$.

From the previous part of the proof we have $w(z)>0$ for all $z \in \Omega$. Hence

$$
m_{\rho}=\min \left[w(z): z \in \partial B_{\rho}(\widehat{z})\right]>0 .
$$

We set $\widetilde{w}=m_{\rho} \widehat{w}$. Then from (3.4) we have

$$
\begin{array}{cl}
-\operatorname{div}(\Theta(z) D \widetilde{w}(z))=0 & \text { in } B_{2 \rho}(\widehat{z}) \backslash \bar{B}_{\rho}(\widehat{z}), \\
\left.\widetilde{w}\right|_{\partial B_{\rho}(\widehat{z})}=m_{\rho}, & \left.\widetilde{w}\right|_{\partial B_{2 \rho}(\widehat{z})}=0 .
\end{array}
$$

The weak comparison principle (see Pucci and Serrin [19]), implies $\widetilde{w} \leq w$ in $B_{2 \rho}(\widehat{z}) \backslash \bar{B}_{\rho}(\widehat{z})$. Moreover, $\widetilde{w}\left(z_{0}\right)=w\left(z_{0}\right)=0$. Hence

$$
\begin{aligned}
\frac{\partial w}{\partial n}\left(z_{0}\right) \leq \frac{\partial \widetilde{w}}{\partial n}\left(z_{0}\right) & =m_{\rho} \frac{\partial \widehat{w}}{\partial n}\left(z_{0}\right)<0 \quad(\text { see }(3.4)), \\
& \Rightarrow w=v-u \in \operatorname{int} C_{+} .
\end{aligned}
$$

Finally by $\widehat{\lambda}_{1}$ we denote the first eigenvalue of the Dirichlet $p$ - Laplacian. We know (see, for example, Gasinski and Papageorgiou [12]) that $\widehat{\lambda}_{1}>0$ and

$$
\widehat{\lambda}_{1}=\inf \left[\frac{\|D u\|_{p}^{p}}{\|u\|_{p}^{p}}: u \in W_{0}^{1, p}(\Omega), u \neq 0\right] .
$$

\section{Positive solutions}

In this section we prove the bifurcation-type theorem describing the dependence of the positive solutions of $(\mathrm{P})_{\lambda}$ on the parameter $\lambda>0$.

The hypotheses on the reaction $f(z, x)$ of $(\mathrm{P})_{\lambda}$, are the following:

$\mathrm{H}(\mathrm{f}) \quad f: \Omega \times \mathbb{R} \rightarrow \mathbb{R}$ is a Caratheodory function such that $f(z, 0)=0$, for almost all $z \in \Omega$ and

(i) for every $\rho>0$, there exists $\alpha_{\rho} \in L^{\infty}(\Omega)_{+}$such that

$$
f(z, x) \leq \alpha_{\rho}(z) \text { for a.a. } z \in \Omega \text {, all } x \in[0, \rho] ;
$$

(ii) $\lim _{x \rightarrow+\infty} \frac{f(z, x)}{x^{p-1}}=0 \quad$ uniformly for almost all $z \in \Omega$;

(iii) $\lim _{x \rightarrow 0^{+}} \frac{f(z, x)}{x^{p-1}}=0 \quad$ uniformly for almost all $z \in \Omega$;

(iv) for every $\rho>0$, there exists $\xi_{\rho}>0$ such that for almost all $z \in \Omega$, $x \rightarrow f(z, x)+\xi_{\rho} x^{p-1}$ is nondecreasing on $[0, \rho]$; 
(v) $f(z, x)>0$ for almost all $z \in \Omega$ and all $x>0$.

REMARK 3.1. Since we are looking for positive solutions and the hypotheses concern only the positive semiaxis $\mathbb{R}_{+}=[0,+\infty$ ), we may (and will) assume that

$$
f(z, x)=0 \quad \text { for a.a. } z \in \Omega \text { and all } x \leq 0 .
$$

Hypothesis $\mathrm{H}(\mathrm{f})$ (ii) implies that for aalmost all $z \in \Omega, f(z, \cdot)$ is strictly $(p-1)$ sublinear near $+\infty$.

EXAMPLE 3.2. Let

$$
g(x)= \begin{cases}x^{r-1} & \text { if } x \in[0,1], \\ x^{q-1} & \text { if } x>1,\end{cases}
$$

with $1<q<p<r<\infty, \alpha \in L^{\infty}(\Omega)_{+}, \alpha(z)>0$ for almost all $z \in \Omega$ and let $f(z, x)=\alpha(z) g(x)$. Then $f(z, x)$ satisfies hypotheses $\mathrm{H}(\mathrm{f})$.

Let $\mathcal{S}=\left\{\lambda>0\right.$ : problem $(\mathrm{P})_{\lambda}$ has a nontrivial positive solution $\}$ and let $S(\lambda)$ be the corresponding solution set of $(\mathrm{P})_{\lambda}$. We set $\lambda_{*}=\inf \mathcal{S}$ (if $\mathcal{S}=\emptyset$, then $\left.\lambda_{*}=+\infty\right)$.

Proposition 3.3. If hypotheses $\mathrm{H}(\mathrm{a}), \mathrm{H}(\mathrm{f})$ hold, then

$$
S(\lambda) \subseteq \operatorname{int} C_{+} \quad \text { and } \quad \lambda_{*}>0 .
$$

Proof. Suppose that $\mathcal{S} \neq \emptyset$ and let $\lambda \in \mathcal{S}$. Then we can find $u \in W_{0}^{1, p}(\Omega)$, $u \geq 0, u \neq 0$ such that

$$
-\operatorname{div} a(D u(z))=\lambda f(z, u(z)) \quad \text { in } \Omega,\left.\quad u\right|_{\partial \Omega}=0 .
$$

From Ladyzhenskaya and Ural'tseva [14, p. 286], we have that $u \in L^{\infty}(\Omega)$. Then invoking the regularity result of Lieberman [17, p. 320], we have that $u \in C_{+} \backslash\{0\}$. Let $\rho=\|u\|_{\infty}$ and let $\xi_{\rho}>0$ be as postulated by hypothesis $\mathrm{H}(\mathrm{f})(\mathrm{iv})$. We have

$$
\begin{aligned}
& -\operatorname{div} a(D u(z))+\lambda \xi_{\rho} u(z)^{p-1}=\lambda f(z, u(z))+\lambda \xi_{\rho} u(z)^{p-1} \geq 0 \quad \text { a.e. in } \Omega, \\
& \Rightarrow \operatorname{div} a(D u(z)) \leq \lambda \xi_{\rho} u(z)^{p-1} \quad \text { a.e. in } \Omega \text {, } \\
& \Rightarrow u \in \operatorname{int} C_{+} \quad \text { (see Pucci-Serrin [19, p. 120]). }
\end{aligned}
$$

So, we have proved that $S(\lambda) \subseteq \operatorname{int} C_{+}$.

Hypotheses $H(f)$ (i), (ii) imply that we can find $c_{6}>0$ such that

$$
f(z, x) \leq c_{6} x^{p-1} \quad \text { for a.a. } z \in \Omega \text {, all } x \geq 0 .
$$

Let $\lambda_{0}<c_{1} \widehat{\lambda}_{1} /\left((p-1) c_{6}\right)$ (see $\left.(2.2)\right)$ and $\eta \in\left(0, \lambda_{0}\right]$. Suppose that $\eta \in \mathcal{S}$. Then by virtue of the first part of the proof, we can find $u_{\eta} \in S(\eta) \subseteq \operatorname{int} C_{+}$. We have

$$
\begin{aligned}
A\left(u_{\eta}\right)=\eta N_{f}\left(u_{\eta}\right), \Rightarrow \frac{c_{1}}{p-1}\left\|D u_{\eta}\right\|_{p}^{p} & \leq \int_{\Omega} \eta f\left(z, u_{\eta}\right) u_{\eta} d z \quad(\text { see Lemma 2.2) } \\
& \leq \eta c_{6}\left\|u_{\eta}\right\|_{p}^{p}<\frac{c_{1}}{p-1} \widehat{\lambda}_{1}\left\|u_{\eta}\right\|_{p}^{p},
\end{aligned}
$$


which contradicts (3.5). Therefore $\eta \notin \mathcal{S}$ and so $\lambda_{*} \geq \lambda_{0}>0$.

For $\lambda>0$, let $\varphi_{\lambda}: W_{0}^{1, p}(\Omega) \rightarrow \mathbb{R}$ be the energy functional for problem $(\mathrm{P})_{\lambda}$ defined by

$$
\varphi_{\lambda}(u)=\int_{\Omega} G(D u) d z-\lambda \int_{\Omega} F(z, u) d z \quad \text { for all } u \in W_{0}^{1, p}(\Omega),
$$

where $F(z, x)=\int_{0}^{x} f(z, s) d s$. Clearly, $\varphi_{\lambda} \in C^{1}\left(W_{0}^{1, p}(\Omega)\right)$.

Proposition 3.4. If hypotheses $\mathrm{H}(\mathrm{a}), \mathrm{H}(\mathrm{f})$ hold, then $\mathcal{S} \neq \emptyset$.

Proof. Hypotheses H(f)(i), (ii), imply that given $\varepsilon>0$, we can find $c_{7}=$ $c_{7}(\varepsilon)>0$ such that

$$
F(z, x) \leq \frac{\varepsilon}{p} x^{p}+c_{7} \quad \text { for a.a. } z \in \Omega, \text { all } x \geq 0 .
$$

Therefore for $u \in W_{0}^{1, p}(\Omega)$, we have

$$
\begin{array}{rlrl}
\varphi_{\lambda}(u) & \geq \frac{c_{1}}{p(p-1)}\|D u\|_{p}^{p}-\frac{\varepsilon}{p}\left\|u^{+}\right\|_{p}^{p}-c_{7}|\Omega|_{N} & & (\text { see Corollary } 2 \text { and }(3.7)) \\
& \geq \frac{1}{p}\left[\frac{c_{1}}{p-1}-\frac{\varepsilon}{\widehat{\lambda}_{1}}\right]\|u\|^{p}-c_{7}|\Omega|_{N} \quad & (\text { see }(3.5)) .
\end{array}
$$

Choosing $\varepsilon \in\left(0, \widehat{\lambda}_{1} c_{1} /(p-1)\right)$, we see that $\varphi_{\lambda}$ is coercive. Also, exploiting the compact embedding of $W_{0}^{1, p}(\Omega)$ into $L^{p}(\Omega)$, we check that $\varphi_{\lambda}$ is sequentially weakly lower semicontinuous. So, by the Weierstrass theorem, we can find $\widehat{u} \in$ $W_{0}^{1, p}(\Omega)$ such that

$$
\varphi_{\lambda}(\widehat{u})=\inf \left[\varphi_{\lambda}(u): u \in W_{0}^{1, p}(\Omega)\right] .
$$

Let $L: L^{p}(\Omega) \rightarrow \mathbb{R}$ be the integral functional defined by

$$
L(v)=\int_{\Omega} F(z, v(z)) d z \quad \text { for all } v \in L^{p}(\Omega) .
$$

By virtue of hypothesis $\mathrm{H}(\mathrm{f})(\mathrm{v})$ we see that for every $v \in L^{p}(\Omega)$ such that $v \geq 0$ and $v \neq 0$, we have that $L(v)>0$. Since the space $W_{0}^{1, p}(\Omega)$ is dense in $L^{p}(\Omega)$, we can find $\widehat{v} \in W_{0}^{1, p}(\Omega), \widehat{v} \geq 0$ such that $L(\widehat{v})>0$. Then we can choose $\lambda>0$ large such that

$$
\lambda L(\widehat{v})>\int_{\Omega} G(D \widehat{v}) d z \Rightarrow \varphi_{\lambda}(\widehat{v})<0 \Rightarrow \varphi_{\lambda}(\widehat{u})<0=\varphi_{\lambda}(0)
$$

(see (3.8)), hence $\widehat{u} \neq 0$. From (3.8), we have

$$
\varphi_{\lambda}^{\prime}(\widehat{u})=0 \Rightarrow A(\widehat{u})=\lambda N_{f}(\widehat{u}) .
$$

Acting on (3.4) with $-\widehat{u}^{-} \in W_{0}^{1, p}(\Omega)$ and using Lemma 2.2, we obtain $\widehat{u} \geq 0$, $\widehat{u} \neq 0$. Therefore $\widehat{u} \in S(\lambda) \subseteq \operatorname{int} C_{+}$for $\lambda>0$ large. Hence $\mathcal{S} \neq \emptyset$.

Proposition 3.5. If hypotheses $\mathrm{H}(\mathrm{a}), \mathrm{H}(\mathrm{f})$ hold and $\lambda \in \mathcal{S}$, then

$$
[\lambda,+\infty) \subseteq \mathcal{S} .
$$


Proof. Let $u_{\lambda} \in S(\lambda) \subseteq \operatorname{int} C_{+}$(see Proposition 3.3). Also let $\theta>\lambda$ and consider the following Caratheodory function:

$$
h_{\theta}(z, x)= \begin{cases}\theta f\left(z, u_{\lambda}(z)\right) & \text { if } x \leq u_{\lambda}(z), \\ \theta f(z, x) & \text { if } u_{\lambda}(z)<x .\end{cases}
$$

We set

$$
H_{\theta}(z, x)=\int_{0}^{x} h_{\theta}(z, s) d s
$$

and then introduce the $C^{1}$-functional $\psi_{\theta}: W_{0}^{1, p}(\Omega) \rightarrow \mathbb{R}$ defined by

$$
\psi_{\theta}(u)=\int_{\Omega} G(D u) d z-\int_{\Omega} H_{\theta}(z, u) d z \quad \text { for all } u \in W_{0}^{1, p}(\Omega) .
$$

As we did for the functional $\varphi_{\lambda}$ in the proof of Proposition 3.4, we show that $\psi_{\theta}$ is coercive and sequentially weakly lower semicontinuous. So, we can find $u_{\theta} \in W_{0}^{1, p}(\Omega)$ such that

$$
\begin{aligned}
\psi_{\theta}\left(u_{\theta}\right)=\inf \left[\psi_{\theta}(u): u \in W_{0}^{1, p}(\Omega)\right] & \Rightarrow \psi_{\theta}^{\prime}\left(u_{\theta}\right)=0 \\
& \Rightarrow A\left(u_{\theta}\right)=N_{h_{\theta}}\left(u_{\theta}\right) .
\end{aligned}
$$

On (3.6) we act with $\left(u_{\lambda}-u_{\theta}\right)^{+} \in W_{0}^{1, p}(\Omega)$. Then

$$
\begin{aligned}
\left\langle A\left(u_{\theta}\right),\left(u_{\lambda}-u_{\theta}\right)^{+}\right\rangle & =\int_{\Omega} h_{\theta}\left(z, u_{\theta}\right)\left(u_{\lambda}-u_{\theta}\right)^{+} d z \\
& =\int_{\Omega} \theta f\left(z, u_{\lambda}\right)\left(u_{\lambda}-u_{\theta}\right)^{+} d z \quad(\text { see }(3.5)) \\
& \geq \int_{\Omega} \lambda f\left(z, u_{\lambda}\right)\left(u_{\lambda}-u_{\theta}\right)^{+} d z \quad(\text { since } \lambda<\theta, f \geq 0) \\
& =\left\langle A\left(u_{\lambda}\right),\left(u_{\lambda}-u_{\theta}\right)^{+}\right\rangle \\
& \Rightarrow \int_{\left\{u_{\lambda}>u_{\theta}\right\}}\left(a\left(D u_{\lambda}\right)-a\left(D u_{\theta}\right), D u_{\lambda}-D u_{\theta}\right)_{\mathbb{R}^{N}} d z \leq 0 \\
& \Rightarrow\left|\left\{u_{\lambda}>u_{\theta}\right\}\right|_{N}=0
\end{aligned}
$$

(see Lemma 2.2), hence $u_{\lambda} \leq u_{\theta}$. Then from (3.5) and (3.6) we have

$$
\begin{aligned}
A\left(u_{\theta}\right)=\theta N_{f}\left(u_{\theta}\right) & \Rightarrow u_{\theta} \in S(\theta) \subseteq \operatorname{int} C_{+} \quad \text { and so } \theta \in \mathcal{S}, \\
& \Rightarrow[\lambda,+\infty) \subseteq \mathcal{S} .
\end{aligned}
$$

From this proposition it follows that $\left(\lambda_{*},+\infty\right) \subseteq \mathcal{S}$.

Proposition 3.6. If hypotheses $\mathrm{H}(\mathrm{a}), \mathrm{H}(\mathrm{f})$ hold and $\lambda>\lambda^{*}$, then problem $(\mathrm{P})_{\lambda}$ has at least two nontrivial positive solutions $u_{0}, \widehat{u} \in \operatorname{int} C_{+}$.

Proof. We know that $\left(\lambda_{*},+\infty\right) \subseteq \mathcal{S}$. Let $\lambda_{*}<\mu<\lambda<\theta$. We can find $u_{\mu} \in S(\mu) \subseteq \operatorname{int} C_{+}$and $u_{\theta} \in S(\theta) \subseteq \operatorname{int} C_{+}$(see Proposition 3.3) and we can have $u_{\mu} \leq u_{\theta}$ (see the proof of Proposition 3.5). 
We introduce the following Caratheodory function

$$
\gamma_{\lambda}(z, x)= \begin{cases}\lambda f\left(z, u_{\mu}(z)\right) & \text { if } x<u_{\mu}(z) \\ \lambda f(z, x) & \text { if } u_{\mu}(z) \leq x \leq u_{\theta}(z), \\ \lambda f\left(z, u_{\theta}(z)\right) & \text { if } u_{\theta}(z)<x\end{cases}
$$

Let

$$
\Gamma_{\lambda}(z, x)=\int_{0}^{x} \gamma_{\lambda}(z, s) d s
$$

and consider the $C^{1}$-functional $\sigma_{\lambda}: W_{0}^{1, p}(\Omega) \rightarrow \mathbb{R}$ defined by

$$
\sigma_{\lambda}(u)=\int_{\Omega} G(D u) d z-\int_{\Omega} \Gamma_{\lambda}(z, u) d z \quad \text { for all } u \in W_{0}^{1, p}(\Omega) .
$$

Clearly $\sigma_{\lambda}$ is coercive (see (3.7)) and sequentially weakly lower semicontinuous. So, we can find $u_{0} \in W_{0}^{1, p}(\Omega)$ such that

$$
\begin{aligned}
\sigma_{\lambda}\left(u_{0}\right)=\inf \left[\sigma_{\lambda}(u): u \in W_{0}^{1, p}(\Omega)\right] & \Rightarrow \sigma_{\lambda}^{\prime}\left(u_{0}\right)=0, \\
& \Rightarrow A\left(u_{0}\right)=N_{\gamma_{\lambda}}\left(u_{0}\right) .
\end{aligned}
$$

Acting on (3.8) first with $\left(u_{\mu}-u_{0}\right)^{+} \in W_{0}^{1, p}(\Omega)$ and then with $\left(u_{0}-u_{\theta}\right)^{+} \in$ $W_{0}^{1, p}(\Omega)$ we show that

$$
\begin{aligned}
u_{0} \in\left[u_{\mu}, u_{\theta}\right]=\left\{u \in W_{0}^{1, p}(\Omega): u_{\mu}(z) \leq u(z) \leq u_{\theta}(z)\right. & \text { a.e. in } \Omega\} \\
& \Rightarrow u_{0} \in S(\lambda) \subseteq \operatorname{int} C_{+}
\end{aligned}
$$

(see (3.7) and (3.8)).

Let $\rho=\left\|u_{\theta}\right\|_{\infty}$ and let $\xi_{\rho}>0$ be as postulated by hypothesis $\mathrm{H}(\mathrm{f})(\mathrm{iv})$. Then

$$
\begin{aligned}
-\operatorname{div} a\left(D u_{\mu}(z)\right)+\mu \xi_{\rho} u_{\mu}(z)^{p-1} & =\mu f\left(z, u_{\mu}(z)\right)+\mu \xi_{\rho} u_{\mu}(z)^{p-1} \\
& \leq \lambda f\left(z, u_{0}(z)\right)+\lambda \xi_{\rho} u_{0}(z)^{p-1}
\end{aligned}
$$

(see $\mathrm{H}(\mathrm{f})(\mathrm{iv})$ and recall $\left.u_{\mu} \leq u_{0}, \mu<\lambda\right)$

$$
\begin{aligned}
& =-\operatorname{div} a\left(D u_{0}(z)\right)+\lambda \xi_{\rho} u_{0}(z)^{p-1} \quad \text { a.e. in } \Omega, \\
& \Rightarrow u_{0}-u_{\mu} \in \operatorname{int} C_{+}
\end{aligned}
$$

(see Proposition 2.5).

In a similar fashion, we show that $u_{\theta}-u_{0} \in \operatorname{int} C_{+}$. So, we have proved that

$$
u_{0} \in \operatorname{int}_{C_{0}^{1}(\bar{\Omega})}\left[u_{\mu}, u_{\theta}\right] .
$$

From (3.5) and (3.7) it follows that

$$
\begin{array}{rlr}
\left.\varphi_{\lambda}\right|_{\left[u_{\mu}, u_{\theta}\right]} & =\left.\sigma_{\lambda}\right|_{\left[u_{\mu}, u_{\theta}\right]}+\beta_{\lambda}^{*} \text { with } \beta_{\lambda}^{*} \in \mathbb{R}, \\
& \left.\Rightarrow u_{0} \text { is a local } C_{0}^{1}(\bar{\Omega}) \text {-minimizer of } \varphi_{\lambda} \quad \text { (see }(3.9)\right) \\
& \Rightarrow u_{0} \text { is a local } W_{0}^{1, p}(\Omega) \text {-minimizer of } \varphi_{\lambda} \quad \text { (see Proposition 2.3). }
\end{array}
$$


Hypothesis $\mathrm{H}(\mathrm{f})(\mathrm{iii})$ implies that given $\varepsilon>0$, we can find $\delta=\delta(\varepsilon)>0$ such that

$$
F(z, x) \leq \frac{\varepsilon}{p} x^{p} \quad \text { for a.a. } z \in \Omega, \text { all } x \in[0, \delta] .
$$

Then, for $u \in C_{0}^{1}(\bar{\Omega})$ with $\|u\|_{C_{0}^{1}(\bar{\Omega})} \leq \delta$, we have

$$
\varphi_{\lambda}(u) \geq \frac{1}{p}\left[\frac{c_{1}}{p-1}-\frac{\lambda \varepsilon}{\widehat{\lambda}_{1}}\right]\|u\|^{p}
$$

(see Corollary 2 and $(3.5),(3.10))$. Choosing $\varepsilon \in\left(0, c_{1} \widehat{\lambda}_{1} /((p-1) \lambda)\right)$, we see that $u=0$ is a local $C_{0}^{1}(\bar{\Omega})$-minimizer of $\varphi_{\lambda}$, hence $u=0$ is a local $W_{0}^{1, p}(\Omega)$-minimizer of $\varphi_{\lambda}$ (see Proposition 2.3).

Therefore we have two local minimizers $0, u_{0}$ of $\varphi_{\lambda}$. Without any loss of generality we may assume that $\varphi_{\lambda}(0)=0 \leq \varphi_{\lambda}\left(u_{0}\right)$ (the analysis is similar if the opposite inequality holds). As in Aizicovici, Papageorgiou and Staicu [1] (see the proof of Proposition 29), we can find $\rho \in(0,1)$ small such that $\left\|u_{0}\right\|>\rho$ and

$$
\varphi_{\lambda}(0)=0 \leq \varphi_{\lambda}\left(u_{0}\right)<\inf \left[\varphi_{\lambda}(u):\left\|u-u_{0}\right\|=\rho\right]=\eta_{\rho}^{\lambda} .
$$

Since $\varphi_{\lambda}$ is coercive, it satisfies the Palais-Smale condition. This fact together with (3.11) permit the application of the mountain pass theorem (see, for example, Gasinski and Papageorgiou [12, p. 648]). So, we can find $\widehat{u} \in W_{0}^{1, p}(\Omega)$ such that

$$
\begin{gathered}
\eta_{\rho}^{\lambda} \leq \varphi_{\lambda}(\widehat{u}), \\
\varphi_{\lambda}^{\prime}(\widehat{u})=0 .
\end{gathered}
$$

From (3.11), (3.12) we have $\widehat{u} \notin\left\{0, u_{0}\right\}$. From (3.13) we have

$$
\widehat{u} \in S(\lambda) \subseteq \operatorname{int} C_{+} .
$$

Proposition 3.7. If hypotheses $\mathrm{H}(\mathrm{a}), \mathrm{H}(\mathrm{f})$ hold, then $\lambda_{*} \in \mathcal{S}$.

Proof. Let $\left\{\lambda_{n}\right\}_{n \geq 1} \subseteq \mathcal{S}$ be a sequence such that

$$
\lambda_{n}>\lambda_{*} \text { for all } n \geq 1 \text { and } \lambda_{n} \downarrow \lambda_{*} \text { as } n \rightarrow \infty \text {. }
$$

We can find $u_{n} \in S\left(\lambda_{n}\right) \subseteq \operatorname{int} C_{+}$such that

$$
A\left(u_{n}\right)=\lambda_{n} N_{f}\left(u_{n}\right) \quad \text { for all } n \geq 1 .
$$

From the proof of Proposition 3.5, we know that we can have

$$
u_{n} \leq u_{1} \text { for all } n \geq 1
$$

From (3.6) we know that

$$
f(z, x) \leq c_{6} x^{p-1} \quad \text { for a.a. } z \in \Omega, \text { all } x \geq 0 .
$$


From (3.14)-(3.16), via Lemma 2.2, we infer that $\left\{u_{n}\right\}_{n \geq 1} \subseteq W_{0}^{1, p}(\Omega)$ is bounded. So, we may assume that

$$
u_{n} \stackrel{w}{\longrightarrow} u_{*} \quad \text { in } W_{0}^{1, p}(\Omega) \quad \text { and } \quad u_{n} \rightarrow u_{*} \quad \text { in } L^{p}(\Omega) .
$$

On (3.14) we act with $u_{n}-u_{*} \in W_{0}^{1, p}(\Omega)$. Passing to the limit as $n \rightarrow \infty$ and using (3.17), we obtain

$$
\lim _{n \rightarrow \infty}\left\langle A\left(u_{n}\right), u_{n}-u_{*}\right\rangle=0 \Rightarrow u_{n} \rightarrow u_{*} \quad \text { in } W_{0}^{1, p}(\Omega)
$$

(see Proposition 2.4). So, if in (3.14) we pass to the limit as $n \rightarrow \infty$, then

$$
A\left(u_{*}\right)=\lambda_{*} N_{f}\left(u_{*}\right) \Rightarrow u_{*} \in C_{+} .
$$

We need to show that $u_{*} \neq 0$. From (3.14) we have

$$
-\operatorname{div} a\left(D u_{n}(z)\right)=\lambda_{n} f\left(z, u_{n}(z)\right) \quad \text { a.e. in } \Omega,\left.\quad u_{n}\right|_{\partial \Omega}=0 .
$$

From Ladyzhenskaya and Ural'tseva [14, p. 286], we know that we can find $M_{1}>0$ such that

$$
\left\|u_{n}\right\|_{\infty} \leq M_{1} \text { for all } n \geq 1
$$

Then invoking the regularity result of Lieberman[17, p. 320], we can find $\beta \in$ $(0,1)$ and $M_{2}>0$ such that

$$
u_{n} \in C_{0}^{1, \beta}(\bar{\Omega}) \quad \text { and } \quad\left\|u_{n}\right\|_{C_{0}^{1, \beta}(\bar{\Omega})} \leq M_{1} \text { for all } n \geq 1 .
$$

Since $C_{0}^{1, \beta}(\bar{\Omega})$ is embedded compactly in $C_{0}^{1}(\bar{\Omega})$, we may assume that $u_{n} \rightarrow u_{*}$ in $C_{0}^{1}(\bar{\Omega})$. Suppose that $u_{*}=0$. Then

$$
u_{n} \rightarrow 0 \quad \text { in } C_{0}^{1}(\bar{\Omega}) .
$$

Hypothesis $\mathrm{H}(\mathrm{f})(\mathrm{iii})$ implies that given $\varepsilon>0$, we can find $\delta=\delta(\varepsilon)>0$ such that

$$
f(z, x) \leq \varepsilon x^{p-1} \quad \text { for a.a. } z \in \Omega \text {, all } x \in[0, \delta] .
$$

From (3.18) we know that we can find $n_{0} \geq 1$ such that

$$
\begin{aligned}
u_{n}(z) & \in[0, \delta] & & \text { for all } z \in \bar{\Omega}, \text { all } n \geq n_{0}, \\
& \Rightarrow-\operatorname{div} a\left(D u_{n}(z)\right) \leq \lambda_{n} \varepsilon u_{n}(z)^{p-1} & & \text { for a.a. } z \in \Omega, \text { all } n \geq n_{0},
\end{aligned}
$$

$(\operatorname{see}(3.19))$

$$
\Rightarrow \frac{c_{1}}{p-1}\left\|D u_{n}\right\|_{p}^{p} \leq \lambda_{n} \varepsilon\left\|u_{n}\right\|_{p}^{p} \leq \frac{\lambda_{n}}{\widehat{\lambda}_{1}} \varepsilon\left\|D u_{n}\right\|_{p}^{p} \quad \text { for all } n \geq n_{0}
$$

(see Lemma 2.2 and (3.5))

$$
\begin{aligned}
& \Rightarrow \frac{c_{1} \widehat{\lambda}_{1}}{(p-1) \varepsilon} \leq \lambda_{n} \quad \text { for all } n \geq n_{0}, \\
& \Rightarrow \frac{c_{1} \widehat{\lambda}_{1}}{(p-1) \varepsilon} \leq \lambda_{*} \text {. }
\end{aligned}
$$


Since $\varepsilon>0$ is arbitrary, we let $\varepsilon \downarrow 0$ and reach a contradiction. Hence

$$
u_{*} \neq 0 \Rightarrow \lambda_{*} \in \mathcal{S} \text {. }
$$

So, summarizing the situation for problem $(\mathrm{P})_{\lambda}$, we can state the following bifurcation-type result.

THEOREM 3.8. If hypotheses $\mathrm{H}(\mathrm{a}), \mathrm{H}(\mathrm{f})$ hold, then there exists $\lambda_{*}>0$ such that:

(a) for every $\lambda>\lambda_{*}$ problem $(\mathrm{P})_{\lambda}$ has at least two nontrivial positive solutions $u_{0}, \widehat{u} \in \operatorname{int} C_{+}$;

(b) for $\lambda=\lambda_{*}$ problem $(\mathrm{P})_{\lambda}$ has at least one positive solution $u_{*} \in \operatorname{int} C_{+}$;

(c) for $\lambda \in\left(0, \lambda_{*}\right)$ problem $(\mathrm{P})_{\lambda}$ has no nontrivial positive solution.

\section{REFERENCES}

[1] S. Aizicovici, N.S. Papageorgiou and V. Staicu, Degree Theory for Operators of Monotone Type and Nonlinear Elliptic Equations with Inequality Constraints, Mem. Amer. Math. Soc., Vol. 196 (2008).

[2] D. Arcoya and D. Ruiz, The Ambrosetti-Prodi problem for the p-Laplace operator, Comm. Partial Differential Equations 31 (2006), 849-865.

[3] V. Benci, P. D'Avenia, D. Fortunato and L. Pisani, Solitons in several space dimensions: Derrick's problem and infinitely many solutions, Arch. Rat. Mech. Anal. 154 (2000), 297-324.

[4] H. Brezis and L. Nirenberg, $H^{1}$ versus $C^{1}$ local minimizers, C.R. Acad. Sci. Paris 317 (1993), 465-472.

[5] F. Brock, L. Itturiaga and P. Ubilla, A multiplicity result for the p-Laplacian involving a parameter, Ann. Inst. H. Poincaré 9 (2008), 1371-1386.

[6] T. Cardinali, N.S. Papageorgiou and P. RubBioni, Bifurcation phenomena for nonlinear superdiffusive Neumann equations of logistic type, Ann. Mat. Pura Appl. 193 (2014), $1-21$.

[7] L. Cherfils And V. ILyASOv, On the stationary solutions of generalized reaction diffusion equations with $p$ and $q$ Laplacian, Comm. Pure Appl. Anal. 1 (2004), 1-14.

[8] S. Cingolani and M. Degiovanni, Nontrivial solutions for $p$-Laplace equations with right hand side having p-linear growth, Comm. Partial Differential Equations 30 (2005), 1191-1203.

[9] J. Garcia Azorero, J. Manfredi and I. Peral Alonso, Sobolev versus Hölder local minimizers and global multiplicity for some quasilinear elliptic equations, Comm. Contemp. Math. 2 (2000), 385-404.

[10] L. Gasinski and N.S. Papageorgiou, Nonsmooth Critical Point Theory and Nonlinear Boundary Value Problems, Chapman Hall/CRC, Boca Raton, 2005.

[11] _ Nonlinear Analysis, Chapman Hall/CRC, Boca Raton, 2006.

[12] _ Multiple solutions for nonlinear coercive problems with a nonhomogeneous differential operator and a nonsmooth potential, Set Valued and Variational Analysis, DOI 10.1007/s.11228-011-01984-4.

[13] S. Hu AND N.S. PAPAGEORGiou, Multiplicity of solutions for parametric p-Laplacian equations with nonlinearity concave near the origin, Tohoku Math. Jour. 62 (2010), 137-162.

[14] O.A. Ladyzhenskaya and N. Ural'tseva, Linear and Quasilinear Elliptic Equations, Academic Press, New York, 1968. 
[15] J. Lewis, Capacity functions in convex rings, Arch. Ration. Mech. Anal. 66 (1977), 201224 .

[16] G. Li AND G. ZhANG, Multiple solutions for the $p$ and $q$ Laplacian problem with critical exponent, Acta Math. Sci. Ser. B 29 (2009), 903-918.

[17] G. Lieberman, The natural generalization of the natural conditions of Ladyzhenskaya and Ural'tseva for elliptic equations, Comm. Partial Differential Equations 16 (1991), 311-361.

[18] K. PererA, Multiple positive solutions for a class of quasilinear boundary value problems, Electron. J. Differential Equations 7 (2003), 1-5.

[19] P. Pucci And J. Serrin, The Maximum Principle, Birkhäuser, Basel, 2007.

[20] M. Sun, Multiplicity of solutions for a class of quasilinear elliptic equations at resonance, J. Math. Anal. Appl. 386 (2012), 661-668.

[21] J. VAZQUeZ, A strong maximum principle for some quasilinear elliptic equations, Appl. Math. Optim. 12 (1984), 191-202.

Nikolaos S. Papageorgiou and George Smyrlis

National Technical University of Athens

Department of Mathematics

Zografou Campus

Athens 157 80, GREECE

E-mail address: npapg@math.ntua.gr, gsmyrlis@math.ntua.gr 